\title{
Evaluation of functional MR Imaging (fMRI) Analysis Methods
}

\author{
Jung-Rung Yang, *Ming-Jang Chiu, **G.M.Huang, J.H.Chen \\ Biomedical Engineering Group,Dept. of Electrical Engineering, National Taiwan University \\ *Department of Neurology, NTU Hospital \\ **Department of Radiology, NTU Hospital
}

\begin{abstract}
In this paper, we design a time series of brain images under activation task and simulate the results of four frequently used fMRI analysis methods. ROC curves for these methods are used to evaluate the method of choice.

Keyword : fMRI, ROC curve

\section{Introduction}

fMRI study has gained its importance in recent years. However, due to the subtle signal change of activation area at commercial MR Imager, typically $3-5 \%$ at $1.5 \mathrm{~T}$, statistical analysis methods must be employed to highlight these associated areas. Similar experiments have been conducted in our lab, yet no conclusive results could be achieved due to its inherent complicated issues, such as inflow effects and misregistration. To make the problem more straight forward, we, therefore, simulate the $\mathrm{AMRI}$ task with given activation areas in brain with different signal changes, mimicking the effects of different field strength, during activation task and evaluate the simulated results using ROC Curve to determine the method of choice.
\end{abstract}

\section{Methods}

To simulate the activation task, we generate 36 time series brain images in a 6-state ( OFF, ON, OFF, ON, OFF, ON) task with 6 images per states. Four different "activation" ROIs with different signal difference-to-noise $\Delta \mathrm{S} / \mathrm{N}$ equals to $0.5,1,2,3$, respectively, are generated to simulate various degrees of activation or the same region at different magnetic field strengths(Fig.1). For a brain image with $S / N$ equals to 20 , these $\Delta \mathrm{S} / \mathrm{N}$ values accounts for real situations for visual cortex at $1.5 \mathrm{~T}$, $2.2 \mathrm{~T}, 3.4 \mathrm{~T}$ and $4 . \mathrm{T}$, respectively[1]. These images are generated with a gaussian distribution random number generator with the ideal exponential curve value as means and certain given values as variances.

Four frequently used AMRI analysis methods in literature are tested. These includes, direct-subtraction method (DS) [2], correlation - coefficient method (CC) $[3,4]$ and statistical-parametrical mapping method (SPM) [5,6]. Direct-subtraction is simply the subtraction of all the baseline images from the active images[2]. Correlation method is to calculate the correlationcoefficient of the acquired temporal results with the given input stimulus[3,4]. To study the effect of different chosen time constant of the square wave inputs, we use two kinds of input stimulus: ideal square wave(CC2) and exponential curve(CC1) which represents the hemodynamic response of the capillaries. SPM method is to estimate the statistical difference between two data groups(active and resting) [5,6].

As for the choice of threshold: Direct -subtraction sets the most significant pixels as "ON" according to the given probability, say, 5\%. In correlation and SPM method, the threshold is determined according to its given probability( say, $5 \%$ ) and image numbers(36) in the F-test and t-test table, respectively.

ROC(Receiver Operating Characteristic) curve is employed to evaluate the performance of each method, where "True" or "False" stands for the fact, "Positive" or "Negative" stands for the results of the analysis method. In ROC curve, sensitivity is plotted against 1-specificity, where,

Sensitivity=True Positive/(True Positive+True Negative)

Specificity=False Negative/(False Positive+False Negative).

Each method will show up as a curve connected by different threshold points. An ideal method should have its sensitivity equals to 1 and 1 - specificity as 0 , shown at the upper-left corner of the ROC curve.

\section{Results and Discussions}

Different image noise levels have been tested in the simulation, our results shows that $\Delta S / N$ is the figure that affects the results. Different noise with the same $\Delta S / N$ yields the same sensitivity and specificity, which is expected. This matches well with the famous Rose model [7].

Among these four methods, the performance of $\mathrm{CCl}$ and SPM methods are about the same and are the best methods(Table 1.), which shown in the upper-left comer of ROC curve(Fig. 2). CC2, with different time constant as the input stimulus in analysis yield slightly worse results. Direct-subtraction, as expected, is the one with the worst performance. These results suggest that, with unknown hemodynamic time constant, SPM should be the method-of-choice. 


\section{Conclusion}

\begin{tabular}{|l|l|l|l|l|l|}
\hline & $P=0.50$ & $P=0.80$ & $P=0.90$ & $P=0.95$ & $P=0.98$ \\
\hline Direct-subtraction & $0.65 / 0.53$ & $0.65 / 0.53$ & $0.35 / 0.83$ & $0.15 / 0.99$ & $0.01 / 0.99$ \\
\hline Correlation-1 & $0.69 / 0.67$ & $0.63 / 0.74$ & $0.59 / 0.77$ & $0.56 / 0.80$ & $0.54 / 0.83$ \\
\hline Correlation-2 & $0.58 / 0.67$ & $0.51 / 0.74$ & $0.47 / 0.77$ & $0.43 / 0.80$ & $0.40 / 0.83$ \\
\hline SPM & $0.81 / 0.48$ & $0.59 / 0.77$ & $0.46 / 0.87$ & $0.33 / 0.93$ & $0.23 / 0.97$ \\
\hline
\end{tabular}

For different $\Delta \mathrm{S} / \mathrm{N}$ ROIs, our results show that the higher the $\Delta S / N$, or equivalently, the higher the field strength, the better the performance(Fig. 3). For $\Delta \mathrm{S} / \mathrm{N}$ higher than 3 , or equally above 4 Tesla, all methods obtain excellent results(Fig. 4).
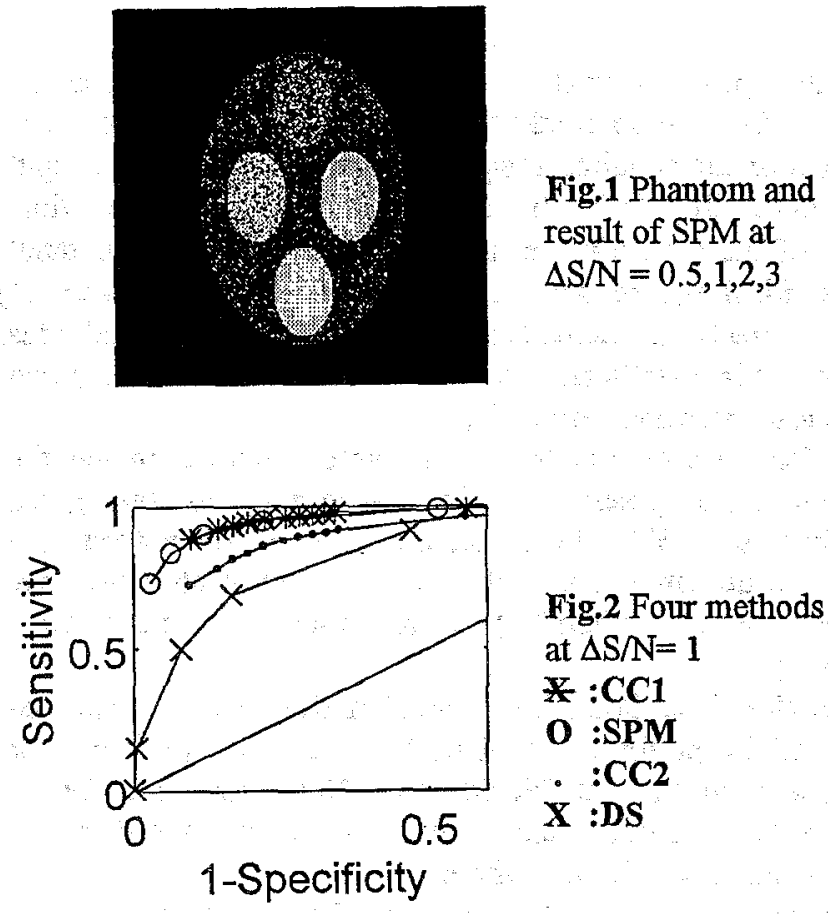

Fig.1 Phantom and result of SPM at $\Delta \mathrm{S} / \mathrm{N}=0.5,1,2,3$

Fig. 2 Four methods at $\Delta \mathrm{S} / \mathrm{N}=1$ * :CC1

0 :SPM . :CC2 1-Specificity

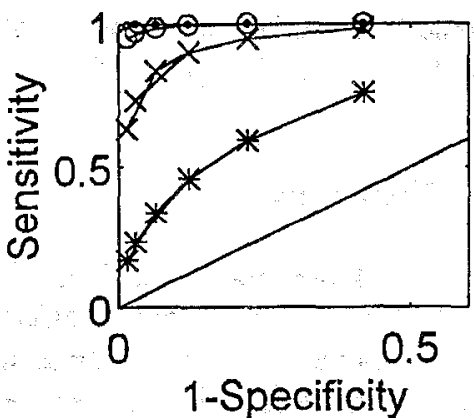

Fig.3 SPM result at $\Delta \mathrm{S} / \mathrm{N}=0.5,1,2,3$

. : $\Delta \mathrm{S} / \mathrm{N}=0.5$

$0: \Delta S / N=1.0$

$\mathrm{X}: \Delta \mathrm{S} / \mathrm{N}=2.0$

: $\Delta \mathrm{S} / \mathrm{N}=3.0$

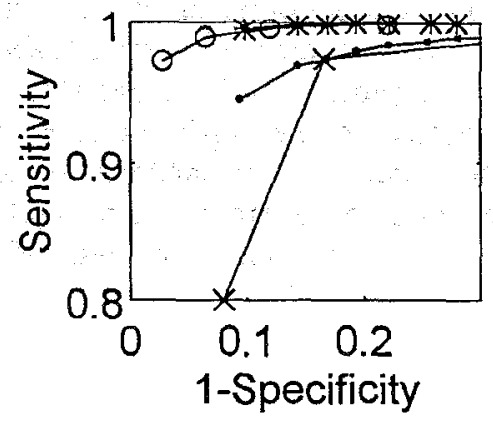

Fig.4 Four methods at $\Delta \mathrm{S} / \mathrm{N}=3.0$

* : CC1

0 : SPM

. : $\mathrm{CC} 2$

$\mathbf{X}: \mathbf{D S}$
Our study shows that SPM is the method of choice for PMRI analysis. With given hemodynamic response of capillaries, correlation coefficient can be as good as SPM method. The performance difference between each method is getting less when magnetic field is higher than about 3.5 to 4 Tesla.

\section{Future Works}

At this moment, we are working on other new analysis methods originated from adaptive signal processing theory. ROC curve of these new methods will be shown in the conference.

\section{Acknowledgment}

This research was financially supported by a grant No. NSC 84-2213-E-002-029 from the National Science Council of ROC.

\section{Reference}

1. Chi-Ming Tsai, Jyh-Horng Chen. Proceedings, 16th IEEE/ EMBS conference, Baltimore, pp. 572-573., 1994

2. O.Henriksen, H.B.W.Larsson, P.Ring, E.Rostrup, Acta Radiologica 34 Fasc. 1,pp. 101-103, 1993.

3. G.K. Wood, B.A. Berkowitz, C.A. Willson, Magn. Reson. Imaging, Vol.12, No. 7 ,P.P. 1013-1020,1994.

4. P.A. Bandettini, A. jesmanowicz, E.C. Wong J.S. Hyde. Magn. Reson. Med. 30, p.p. 161-173, 1993.

5. K.J. Friston, P. Jezzard, and R.Tumer, Human Brain Mapping 1:p.p. 153-171;1994.

6. F. Wenz, L.R. Schad, M.V.Knopp, K.T. Bandendistel, F. Flomer, J. Schroder, G.van Kaick, Magn. Reson. Imaging, Vol:12,No.7, P.P. 1013-1020,1994.

7. A.A. Rose, Vision: Human and Electronic., Plenum Press, NY, 1973. 